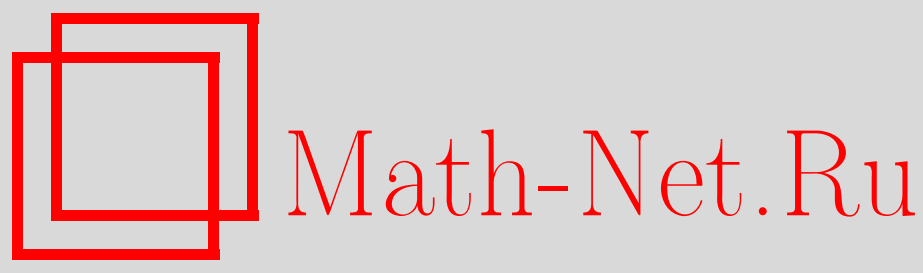

С. М. Ташпулатов, О спектре оператора энергии двухмагнонных систем в трехмерной изотропной примесной ферромагнитной модели Гейзенберга, ТМФ, 2010, том 162, номер 2, 227-242

DOI: https://doi.org/10.4213/tmf6466

Использование Общероссийского математического портала Math-Net.Ru подразумевает, что вы прочитали и согласны с пользовательским соглашением http: //www . mathnet.ru/rus/agreement

Параметры загрузки:

IP : 3.81 .55 .215

26 апреля 2023 г., 15:25:01

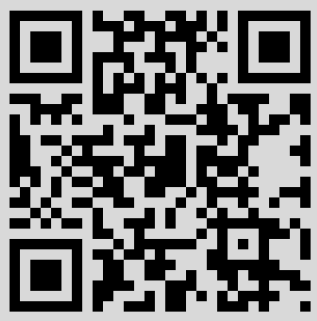




\section{О СПЕКТРЕ ОПЕРАТОРА ЭНЕРГИИ ДВУХМАГНОННЫХ СИСТЕМ В ТРЕХМЕРНОЙ ИЗОТРОПНОЙ ПРИМЕСНОЙ ФЕРРОМАГНИТНОЙ МОДЕЛИ ГЕЙЗЕНБЕРГА}

Рассмотрен оператор энергии двухмагнонных систем в трехмерной изотропной ферромагнитной примесной модели Гейзенберга со взаимодействием ближайших соседей. Исследованы структура существенного спектра и дискретный спектр системы в трехмерной решетке. Показано, что существенный спектр системы состоит из объединения не более чем четырех отрезков, и на краю существенного спектра дискретный спектр системы конечен.

Ключевые слова: дискретный спектр, существенный спектр, модель Гейзенберга, решетка, двухмагнонная система, локальное примесное состояние.

\section{1. ВВЕДЕНИЕ}

Одним из замечательных результатов спектрального анализа трехчастичного непрерывного оператора Шредингера является эффект Ефимова: если в системе трех частиц, взаимодействующих с помощью парных короткодействующих потенциалов, ни одна из трех двухчастичных подсистем не имеет связанных состояний (CC) с отрицательной энергией, но по меньшей мере две из них имеют резонанс с энергией в нуле, то у этой трехчастичной системы существует бесконечное число трехчастичных СС с отрицательной энергией, накапливающихся к нулю. Этот эффект впервые был обнаружен Ефимовым [1]. В работах [2], [3] эта задача изучалась в физическом аспекте. Строгое математическое доказательство существования эффекта Ефимова было проведено впервые в работе [4], а затем в работах [5]-[7].

В моделях физики твердого тела возникает так называемый дискретный оператор Шредингера, являющийся решетчатым аналогом обычного непрерывного трехчастичного оператора Шредингера. Для таких операторов существование эффекта Ефимова на физическом уровне строгости было доказано в работах [8]-[10].

* Институт ядерной физики АНРУз, Ташкент, Узбекистан.

E-mail: toshpul@mail.ru, toshpul@rambler.ru 
В работах Лакаева и его учеников вопрос о существовании эффекта Ефимова рассматривался для решетчатых трехчастичных систем бозонов, взаимодействующих с помощью парных контактных потенциалов притяжения при фиксированном значении полного квазиимпульса системы (см., например, работы [11]-[14]).

В конкретных физических системах доказательство существования эффекта Ефимова или его отсутствия является одной из важнейших задач математической физики.

В настоящей работе рассматривается двухмагнонная система в изотропной примесной ферромагнитной модели Гейзенберга на трехмерной решетке $\mathbb{Z}^{3}$ со взаимодействием ближайших соседей и исследуются существенный и дискретный спектры системы.

Гамильтониан рассматриваемой системы имеет вид

$$
H=J \sum_{m, \tau}\left(\vec{S}_{m} \vec{S}_{m+\tau}\right)+\left(J_{0}-J\right) \sum_{\tau}\left(\vec{S}_{0} \vec{S}_{\tau}\right),
$$

где $J<0$ и $J_{0} \neq 0$ - параметры билинейного обменного взаимодействия между атомами и между атомами и примесями соответственно, $\vec{S}_{m}=\left(S_{m}^{x} ; S_{m}^{y} ; S_{m}^{z}\right)$ - оператор атомного спина величины $s=1 / 2$ в узле $m$, а по $\tau$ ведется суммирование по ближайшим соседям. Гамильтониан (1) действует в симметричном фоковском пространстве $\mathscr{H}$. Обозначим через $\varphi_{0}$ вектор, называемый вакуумным и однозначно определяемый условиями $S_{m}^{+} \varphi_{0}=0, S_{m}^{z} \varphi_{0}=\varphi_{0} / 2$, где $\left\|\varphi_{0}\right\|=1$. Положим $S_{m}^{ \pm}=S_{m}^{x} \pm i S_{m}^{y}$, где $S_{m}^{-}$и $S_{m}^{+}$- операторы рождения и уничтожения магнона в узле $m$ соответственно. Вектор $S_{m}^{-} S_{n}^{-} \varphi_{0}$ описывает состояние системы двух магнонов, находящихся в узлах $m$ и $n$ со значением спина $s=1 / 2$. Векторы $S_{m}^{-} S_{n}^{-} \varphi_{0}$ образуют ортонормальную систему. Пространство, натянутое на них, обозначим через $\widehat{\mathscr{H}}_{2}$. Оно является евклидовым относительно исходного скалярного произведения. Замыкание этого пространства обозначим через $\mathscr{H}_{2}$. Оно называется пространством двухмагнонных состояний оператора $H$.

ПрЕДЛОЖЕНИЕ 1. Пространство $\mathscr{H}_{2}$ инвариантно относительно оператора $H$. Oператор $\mathrm{H}_{2}=\left.H\right|_{\mathscr{H}_{2}}$ является ограниченным самосопряженным оператором, который порождает ограниченный самосопряженный оператор $\bar{H}_{2}$, действующий в пространстве $l_{2}\left(\mathbb{Z}^{3} \times \mathbb{Z}^{3}\right)$ по формуле

$$
\begin{aligned}
\left(\bar{H}_{2} f\right)(p ; q)=J & \sum_{p, q, \tau}\left\{\left[\delta_{p, q+\tau}+\delta_{p+\tau, q}-2\right] f(p ; q)-\frac{1}{2} \delta_{p-\tau, q} f(p-\tau ; q)-\right. \\
& -\frac{1}{2} \delta_{p, q-\tau} f(p ; q-\tau)+\frac{1}{2} f(p ; q-\tau)-\frac{1}{2} \delta_{p+\tau, q} f(p+\tau ; q)+ \\
& \left.+\frac{1}{2} f(p+\tau ; q)+\frac{1}{2} f(p-\tau ; q)+\frac{1}{2} f(p ; q+\tau)-\frac{1}{2} \delta_{p ; q+\tau} f(p ; q+\tau)\right\}+ \\
& +\left(J_{0}-J\right) \sum_{p, q, \tau}\left\{\left[\delta_{p, \tau} \delta_{q, 0}+\delta_{p, 0} \delta_{q, \tau}-\frac{1}{2} \delta_{p, \tau}-\frac{1}{2} \delta_{q, \tau}-\right.\right. \\
& \left.-\frac{1}{2} \delta_{p, 0}-\frac{1}{2} \delta_{q, 0}\right] f(p ; q)-\frac{1}{2} \delta_{p, 0} \delta_{q, \tau} f(p ; 0)-
\end{aligned}
$$




$$
\begin{aligned}
& -\frac{1}{2} \delta_{p, \tau} \delta_{q, 0} f(0 ; q)+\frac{1}{2} \delta_{p, \tau} f(0 ; q)+\frac{1}{2} \delta_{q, \tau} f(p ; 0)-\frac{1}{2} \delta_{p, 0} \delta_{q, \tau} \times \\
& \left.\times f(\tau ; q)-\frac{1}{2} \delta_{p, \tau} \delta_{q, 0} f(p ; \tau)+\frac{1}{2} \delta_{p, 0} f(\tau ; q)+\frac{1}{2} \delta_{q, 0} f(p ; \tau)\right\},
\end{aligned}
$$

где $\delta_{k, j}-$ символ Кронекера. Сам оператор $\mathrm{H}_{2}$ действует на вектор $\psi \in \mathscr{H}_{2}$ по формуле

$$
H_{2}=\sum_{p, q, \tau}\left(\bar{H}_{2} f\right)(p ; q) S_{p}^{-} S_{q}^{-} \varphi_{0} .
$$

ДокАЗАтЕЛЬство. Заметим, что вектор $S_{p}^{-} S_{q}^{-} \varphi_{0}$ под действием оператора $H$ переходит в вектор

$$
\begin{aligned}
H\left(S_{p}^{-} S_{q}^{-} \varphi_{0}\right)=J & \sum_{p, q} \sum_{m, \tau}\left[\left(\delta_{m+\tau, p} \delta_{m, q}+\delta_{m, p} \delta_{m+\tau, q}-\frac{1}{2} \delta_{m, p}-\frac{1}{2} \delta_{m, q}-\right.\right. \\
& \left.-\frac{1}{2} \delta_{m+\tau, p}-\frac{1}{2} \delta_{m+\tau, q}\right) S_{p}^{-} S_{q}^{-} \varphi_{0}-\frac{1}{2} \delta_{m, p} \delta_{m, q} S_{m+\tau}^{-} S_{q}^{-} \varphi_{0}- \\
& -\frac{1}{2} \delta_{m, p} \delta_{m, q} S_{m+\tau}^{-} S_{p}^{-} \varphi_{0}-\frac{1}{2} \delta_{m+\tau, p} \delta_{m+\tau, q} S_{m}^{-} S_{q}^{-} \varphi_{0}- \\
& -\frac{1}{2} \delta_{m+\tau, p} \delta_{m+\tau, q} S_{m}^{-} S_{p}^{-} \varphi_{0}+\frac{1}{2} \delta_{m, p} S_{m+\tau}^{-} S_{q}^{-} \varphi_{0}+ \\
& \left.+\frac{1}{2} \delta_{m, q} S_{m+\tau}^{-} S_{p}^{-} \varphi_{0}+\frac{1}{2} \delta_{m+\tau, p} S_{m}^{-} S_{q}^{-} \varphi_{0}+\frac{1}{2} \delta_{m+\tau, q} S_{m}^{-} S_{p}^{-} \varphi_{0}\right] \\
& +\left(J_{0}-J\right) \sum_{p, q} \sum_{\tau}\left[\left(\delta_{\tau, p} \delta_{0, q}+\delta_{0, p} \delta_{\tau, q}-\frac{1}{2} \delta_{0, p}-\frac{1}{2} \delta_{0, q}-\right.\right. \\
& \left.+\frac{1}{2} \delta_{\tau, p} S_{0}^{-} S_{q}^{-} \varphi_{0}+\frac{1}{2} \delta_{\tau, q} S_{0}^{-} S_{p}^{-} \varphi_{0}\right] . \\
& \left.-\frac{1}{2} \delta_{\tau, p}-\frac{1}{2} \delta_{\tau, q}\right) S_{p}^{-} S_{q}^{-} \varphi_{0}-\frac{1}{2} \delta_{0, p} \delta_{0, q} S_{\tau}^{-} S_{q}^{-} \varphi_{0}- \\
& -\frac{1}{2} \delta_{0, p} \delta_{0, q} S_{\tau}^{-} S_{p}^{-} \varphi_{0}-\frac{1}{2} \delta_{\tau, p} \delta_{\tau, q} S_{0}^{-} S_{q}^{-} \varphi_{0}- \\
& S_{0}^{-} S_{p}^{-} \varphi_{0}+\frac{1}{2} \delta_{0, p} S_{\tau}^{-} S_{q}^{-} \varphi_{0}+\frac{1}{2} \delta_{0, q} S_{\tau}^{-} S_{p}^{-} \varphi_{0}+ \\
& \\
& \\
& \\
& \\
&
\end{aligned}
$$

Это означает инвариантность подпространства $\mathscr{H}_{2}$ относительно оператора $H$.

Далее, оператор $H$ на $\mathscr{H}_{2}$ симметричен, и из формулы (4) и суммируемости $\tau$ по ближайшим соседям следует, что $H_{2}^{\star}=H_{2}$. Следовательно, $H_{2}$ - самосопряженный оператор. Из формулы (4) следует, что оператор $H_{2}$ действует на вектор $\psi \in \mathscr{H}_{2}$ по формуле $(3)$, где $\left(\bar{H}_{2} f\right)(p ; q)$ определяется с помощью формулы $(2)$. 
Покажем, что оператор $\bar{H}_{2}$ является ограниченным. Пусть $F=(f(p ; q))_{p, q \in \mathbb{Z}^{3}}-$ элемент из $l_{2}\left(\mathbb{Z}^{3} \times \mathbb{Z}^{3}\right)$, тогда $\|F\|=\sqrt{\sum_{p, q}|f(p ; q)|^{2}}$ и $\left\|\bar{H}_{2} F\right\|=\sqrt{\left[\left(\bar{H}_{2} f\right)(p ; q)\right]^{2}} \leqslant$ $C_{3}\|F\|$, где $C_{3}=48\left\{|J|+\left|J_{0}-J\right|\right\}$. Ограниченность оператора $H_{2}$ следует из формулы (3). Заметим также, что сопряженный оператор $\left(\bar{H}_{2}^{\star}\right)(p ; q)$ действует в $l_{2}\left(\mathbb{Z}^{3} \times \mathbb{Z}^{3}\right)$ по формуле

$$
\begin{aligned}
\left(\bar{H}_{2}^{\star} f\right)(p ; q)=J & \sum_{p, q, \tau}\left\{\left[\delta_{p, q-\tau}+\delta_{p-\tau, q}-2\right] f(p ; q)-\frac{1}{2} \delta_{p+\tau, q} f(p+\tau ; q)-\right. \\
& -\frac{1}{2} \delta_{p, q+\tau} f(p ; q+\tau)+\frac{1}{2} f(p ; q+\tau)-\frac{1}{2} \delta_{p-\tau, q} f(p-\tau ; q)+ \\
& \left.+\frac{1}{2} f(p-\tau ; q)+\frac{1}{2} f(p+\tau ; q)+\frac{1}{2} f(p ; q-\tau)-\frac{1}{2} \delta_{p ; q-\tau} f(p ; q-\tau)\right\}+ \\
& +\left(J_{0}-J\right) \sum_{p, q, \tau}\left\{\left[\delta_{p, \tau} \delta_{q, 0}+\delta_{p, 0} \delta_{q, \tau}-\frac{1}{2} \delta_{p, \tau}-\frac{1}{2} \delta_{q, \tau}-\right.\right. \\
& \left.-\frac{1}{2} \delta_{p, 0}-\frac{1}{2} \delta_{q, 0}\right] f(p ; q)-\frac{1}{2} \delta_{p, 0} \delta_{q, \tau} f(p ; 0)-\frac{1}{2} \delta_{p, \tau} \delta_{q, 0} f(0 ; q)+ \\
& +\frac{1}{2} \delta_{p, \tau} f(0 ; q)+\frac{1}{2} \delta_{q, \tau} f(p ; 0)-\frac{1}{2} \delta_{p, 0} \delta_{q, \tau} f(\tau ; q)-\frac{1}{2} \delta_{p, \tau} \delta_{q, 0} f(p ; \tau)+ \\
& \left.+\frac{1}{2} \delta_{p, 0} f(\tau ; q)+\frac{1}{2} \delta_{q, 0} f(p ; \tau)\right\} \equiv\left(\bar{H}_{2} f\right)(p ; q) .
\end{aligned}
$$

Следовательно, оператор $\bar{H}_{2}$ симметричен и определен всюду в пространстве $\mathscr{H}_{2}$, поэтому он является самосопряженным.

Лемма 1. Спектры операторов $\mathrm{H}_{2} u \bar{H}_{2}$ совпадают.

ДокАзАТЕЛЬСтво. Так как операторы $H_{2}$ и $\bar{H}_{2}$ являются ограниченными самосопряженными операторами, то из критерия Вейля следует существование последовательности векторов $\psi_{n}$ таких, что

$$
\psi_{n}=\sum_{p, q} f_{n}(p ; q) S_{p}^{-} S_{q}^{-} \varphi_{0}, \quad\left\|\psi_{n}\right\|=1,
$$

И

$$
\lim _{n \rightarrow \infty}\left\|H_{2} \psi_{n}-\lambda \psi_{n}\right\|=0,
$$

где $\lambda \in \sigma\left(H_{2}\right)$. С другой стороны,

$$
\begin{aligned}
\left\|H_{2} \psi_{n}-\lambda \psi_{n}\right\|^{2} & =\left(H_{2} \psi_{n}-\lambda \psi_{n}, H_{2} \psi_{n}-\lambda \psi_{n}\right)= \\
& =\sum_{p, q} \mid\left(\bar{H}_{2} f_{n}(p ; q)-\left.\lambda f_{n}(p ; q)\right|^{2}=\left\|H_{2} F_{n}-\lambda F_{n}\right\|^{2}\right.
\end{aligned}
$$


и

$$
\left\|F_{n}\right\|^{2}=\sum_{p, q}\left|f_{n}(p ; q)\right|^{2}=\left\|\psi_{n}\right\|^{2}=1 .
$$

Отсюда и из формулы (5) при $n \rightarrow \infty$ имеем, что $\left\|H_{2} F_{n}-\lambda F_{n}\right\| \rightarrow 0$ и $F_{n}=$ $\left(f_{n}(p ; q)\right)_{p, q \in \mathbb{Z}^{3}}$. Это означает, что $\lambda \in \sigma\left(\bar{H}_{2}\right)$. Следовательно, $\sigma\left(H_{2}\right) \subset \sigma\left(\bar{H}_{2}\right)$. Обратно, пусть $\bar{\lambda} \in \sigma\left(\bar{H}_{2}\right)$. Тогда в силу того же критерия Вейля существует последовательность $F_{n}$ такая, что $\left\|F_{n}\right\|=\sqrt{\sum_{p, q}\left|f_{n}(p ; q)\right|^{2}}=1$ и

$$
\|\left(\bar{H}_{2} F_{n}-\bar{\lambda} F_{n} \| \rightarrow 0\right.
$$

при $n \rightarrow \infty$.

Полагая $\psi_{n}=\sum_{p, q} f_{n}(p ; q) S_{p}^{-} S_{q}^{-} \varphi_{0}$, имеем $\left\|\psi_{n}\right\|=\left\|F_{n}\right\|=1$ и $\left\|\bar{H}_{2} F_{n}-\bar{\lambda} F_{n}\right\|=$ $\left\|H_{2} \psi_{n}-\bar{\lambda} \psi_{n}\right\|$. Отсюда, из формулы (6) и критерия Вейля вытекает, что $\bar{\lambda} \in \sigma\left(H_{2}\right)$. Следовательно, $\sigma\left(\bar{H}_{2}\right) \subset \sigma\left(H_{2}\right)$.

Наша цель состоит в изучении спектра оператора $H_{2}$. Это удобно сделать в его квазиимпульсном представлении. Обозначим через $\mathscr{F}$ преобразование Фурье:

$$
\mathscr{F}: l_{2}\left(\mathbb{Z}^{3} \times \mathbb{Z}^{3}\right) \Rightarrow L_{2}\left(T^{3} \times T^{3}\right) \text {. }
$$

Здесь $T^{3}$ - трехмерный тор, снабженный нормированной мерой Лебега $d \lambda: \lambda\left(T^{3}\right)=1$.

Оператор $H_{2}$ в квазиимпульсном представлении в пространстве $L_{2}\left(T^{3} \times T^{3}\right)$ действует по формуле

$$
\begin{aligned}
\left(\widetilde{H}_{2} f\right)(x ; y)=[ & \left.-12 J+2 J \sum_{i=1}^{3}\left(\cos x_{i}+\cos y_{i}\right)\right] f(x ; y)- \\
& -\varepsilon \int_{T^{3}}\left\{3+\sum_{i=1}^{3}\left[\cos \left(x_{i}-s_{i}\right)-\cos x_{i}-\cos s_{i}\right]\right\} f(s ; y) d s- \\
& -\varepsilon \int_{T^{3}}\left\{3+\sum_{i=1}^{3}\left[\cos \left(y_{i}-t_{i}\right)-\cos y_{i}-\cos t_{i}\right]\right\} f(x ; t) d t+ \\
& +J \int_{T^{3}}\left\{12-\sum_{i=1}^{3}\left[\cos s_{i}+\cos x_{i}+\cos y_{i}+\right.\right. \\
& \left.\left.+\cos \left(x_{i}+y_{i}-s_{i}\right)\right]\right\} f(s ; x+y-s) d s+ \\
& +\varepsilon \int_{T^{3}}\left\{\int _ { T ^ { 3 } } \left\{6+2 \sum_{i=1}^{3}\left[\cos \left(x_{i}-s_{i}\right)+\cos \left(y_{i}-t_{i}\right)+\right.\right.\right. \\
& \left.+\cos \left(x_{i}+y_{i}-s_{i}-t_{i}\right)\right]-\cos t_{i}-\cos s_{i}-\cos x_{i}-\cos y_{i}- \\
& -\cos \left(x_{i}-s_{i}-t_{i}\right)-\cos \left(y_{i}-s_{i}-t_{i}\right)-\cos \left(x_{i}+y_{i}-t_{i}\right)- \\
& \left.-\cos \left(x_{i}+y_{i}-s_{i}\right)\right\} f(s ; t) d s d t .
\end{aligned}
$$

Здесь $\varepsilon=J_{0}-J$. 
Конечность или бесконечность дискретного спектра данного трехчастичного дискретного оператора Шредингера зависит от количества двухчастичных подсистем, имеющих так называемый виртуальный уровень на краю существенного спектра трехчастичной системы [5]-[7]. Дискретный спектр системы конечен, если на краю существенного спектра только одна двухчастичная подсистема имеет виртуальный уровень или виртуальных уровней у двухчастичных подсистем нет вообще. Если же две или все три двухчастичные подсистемы имеют виртуальные уровни на краю существенного спектра, то дискретный спектр системы бесконечен. Двухчастичные подсистемы данной трехчастичной системы состоят из двух одномагнонных примесных двухчастичных подсистем и системы двух магнонов. Одномагнонные примесные подсистемы неразличимы, поэтому если одна из таких подсистем имеет виртуальный уровень, то другая также имеет виртуальный уровень на краю существенного спектра трехчастичной системы. Следовательно, необходимо сначала изучить спектр двухчастичной подсистемы рассматриваемой трехчастичной системы. Поэтому опишем сначала спектр и локализованные примесные состояния (ЛПС) одномагнонных примесных подсистем, а также спектр и СС двухмагнонных подсистем.

\section{2. ОДНОМАГНОННЫЕ ПРИМЕСНЫЕ СОСТОЯНИЯ}

Интенсивное развитие физики пленочного состояния, а также применение пленок в различных областях физики и техники обусловливают значительный интерес к изучению ЛПС магнетиков.

ЛПС в ферромагнетике Гейзенберга с ферромагнитными или антиферромагнитными примесями изучались во многих работах (см., например, [15]-[20]), где подробно рассматривались случаи линейной или кубической решетки. Было показано, что в линейном случае существует два типа ЛПС, а в кубической решетке - три. В работе [21] исследовались спектр и ЛПС одномагнонных систем в примесной модели Гейзенберга на $\nu$-мерной решетке $\mathbb{Z}^{\nu}$, при этом рассматривался как случай ферромагнетика с ферромагнитной примесью, так и случай ферромагнетика с антиферромагнитной примесью. Показано, что в $\nu$-мерном случае система имеет не более трех (без учета кратности вырождений их энергии) ЛПС, и найдено условие существования этих состояний, а также определено местоположение их энергий относительно непрерывного спектра системы.

Гамильтониан одномагнонной примесной системы также имеет вид (1). Вектор $S_{m}^{-} \varphi_{0}$ описывает состояние системы одного магнона, находящегося в узле $m$, со значением спина $s=1 / 2$. Векторы $S_{m}^{-} \varphi_{0}$ образуют ортонормальную систему. Пространство, натянутое на эти векторы, обозначим через $\widehat{\mathscr{H}}_{1}$. Оно является евклидовым относительно естественного скалярного произведения. Замыкание этого пространства обозначим через $\mathscr{H}_{1}$. Оно называется пространством одномагнонных состояний оператора $H$. 
ПреДЛОЖЕНИЕ 2. Пространство $\mathscr{H}_{1}$ инвариантно относительно оператора $H$. Оператор $H_{1}=\left.H\right|_{\mathscr{H}_{1}}$ является ограниченным самосопряженным оператором, который порождает ограниченный самосопряюенный оператор $\bar{H}_{1}$, действующий в пространстве $l_{2}\left(\mathbb{Z}^{3}\right)$ по формуле

$$
\begin{aligned}
\left(\bar{H}_{1} f\right)(p)=\sum_{p, \tau} & {\left[-J f(p)+\frac{J}{2}(f(p+\tau)+f(p-\tau))\right]+} \\
& +\left(J_{0}-J\right) \sum_{p, \tau}\left[\left(-\delta_{p, 0}-\delta_{p, \tau}\right) f(p)+\left(\delta_{p, \tau} f(0)+\delta_{p, 0} f(\tau)\right)\right]
\end{aligned}
$$

где $\delta_{k, j}$ - символ Кронекера. Сам оператор $H_{1}$ действует на вектор $\psi \in \mathscr{H}_{1}$ по формуле

$$
H_{1} \psi=\sum_{p}\left(\bar{H}_{1} f\right)(p) S_{p}^{-} \varphi_{0}
$$

Предложение 2 доказывается аналогично предложению 1 с использованием известных коммутационных соотношений между операторами $S_{m}^{+}, S_{p}^{-}$и $S_{q}^{z}$.

Лемма 2. Cпектри операторов $H_{1} u \bar{H}_{1}$ совпадают.

ДокАЗАТЕльство леммы аналогично доказательству леммы 1.

Опишем теперь спектр оператора $H_{1}$. Обозначим через $\mathscr{F}$ преобразование Фурье

$$
\mathscr{F}: l_{2}\left(\mathbb{Z}^{3}\right) \Rightarrow L_{2}\left(T^{3}\right) .
$$

ПРЕДЛОЖЕНИЕ 3. Onератор $\bar{H}_{1}$ nepexoдum в оператор $\widetilde{H}_{1}=\mathscr{F} \bar{H}_{1} \mathscr{F}^{-1}$, который действует в пространстве $L_{2}\left(T^{3}\right)$ по формуле

$$
\left(\widetilde{H}_{1} f\right)(x)=h(x) f(x)+\int_{T^{3}} h_{1}(x ; t) f(t) d t
$$

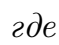

$$
h(x)=-2 J\left[3-\sum_{i=1}^{3} \cos x_{i}\right], \quad h_{1}(x ; t)=-2 \varepsilon\left[3+\sum_{i=1}^{3}\left[\cos \left(x_{i}-t_{i}\right)-\cos x_{i}-\cos t_{i}\right]\right],
$$

$\varepsilon=J_{0}-J ; x, t \in T^{3}$.

ДокАзАТЕЛЬСТво проводится непосредственно с использованием преобразования Фурье.

Известно, что непрерывный спектр оператора $\widetilde{H}_{1}$ не зависит от функции $h_{1}(x ; t)$ и заполняет весь отрезок $\left[m_{3} ; M_{3}\right]$, где $m_{3}=\min _{x \in T^{3}} h(x), M_{3}=\max _{x \in T^{3}} h(x)$.

ОПрЕДЕЛЕниЕ 1 . Собственная функция $\varphi \in L_{2}\left(T^{3}\right)$ оператора $\widetilde{H}_{1}$, отвечающая собственному значению $z$, называется ЛПС оператора $\widetilde{H}_{1}$, а величина $z$ - энергией этого состояния. 
Положим

$$
\Delta_{3}(z)=\left[1-2 \varepsilon J_{3,1}(z)\right]\left[1-2 \varepsilon J_{3,2}(z)\right]^{3}\left[1-\varepsilon J_{3,3}(z)\right]^{2},
$$

где

$$
\begin{aligned}
& J_{3,1}(z)=\int_{T^{3}} \frac{\left(1-\cos s_{1}\right)\left(3-\sum_{i=1}^{3} \cos s_{i}\right) d s_{1} d s_{2} d s_{3}}{h\left(s_{1} ; s_{2} ; s_{3}\right)-z}, \\
& J_{3,2}(z)=\int_{T^{3}} \frac{\sin ^{2} s_{1} d s_{1} d s_{2} d s_{3}}{h\left(s_{1} ; s_{2} ; s_{3}\right)-z} \\
& J_{3,3}(z)=\int_{T^{3}} \frac{\left(\cos s_{1}-\cos s_{2}\right)^{2} d s_{1} d s_{2} d s_{3}}{h\left(s_{1} ; s_{2} ; s_{3}\right)-z} .
\end{aligned}
$$

Лемма 3. Число $z_{0} \notin\left[m_{3} ; M_{3}\right]$ является собственным значением оператора $\widetilde{H}_{1}$ тогда и только тогда, когда оно является нулем функиии $\Delta_{3}(z)$.

ДокАЗАтельство. Уравнение для собственных значений является интегральным уравнением с вырожденным ядром. Поэтому оно эквивалентно системе линейных однородных алгебраических уравнений. Известно, что такая система имеет нетривиальное решение тогда и только тогда, когда детерминант системы равен нулю. Учитывая, что функция $h\left(s_{1} ; s_{2} ; s_{3}\right)$ является симметричной относительно $s_{i}$ и $s_{j}$, и выполняя соответствующие преобразования, получаем, что детерминант системы имеет вид $\Delta_{3}(z)$.

Обозначим через $P$ пару $(J ; \varepsilon)$ и положим

$$
\begin{aligned}
a & =\int_{T^{3}} \frac{\sin ^{2} s_{1} d s_{1} d s_{2} d s_{3}}{3-\cos s_{1}-\cos s_{2}-\cos s_{3}}=\int_{T^{3}} \frac{\sin ^{2} s_{1} d s_{1} d s_{2} d s_{3}}{3+\cos s_{1}+\cos s_{2}+\cos s_{3}}, \\
b & =\int_{T^{3}} \frac{\left(\cos s_{1}-\cos s_{2}\right)^{2} d s_{1} d s_{2} d s_{3}}{3-\cos s_{1}-\cos s_{2}-\cos s_{3}}=\int_{T^{3}} \frac{\left(\cos s_{1}-\cos s_{2}\right)^{2} d s_{1} d s_{2} d s_{3}}{3+\cos s_{1}+\cos s_{2}+\cos s_{3}} .
\end{aligned}
$$

Ясно, что $0<a<b<1$ и $2 a<b$. При $\nu=3$ введем следующие множества пар $P$ :

$$
\begin{aligned}
Q_{1} & =\{P: J<0,0<\varepsilon \leqslant-J\}, & Q_{2} & =\left\{P: J<0, \frac{J}{3}<\varepsilon<0\right\}, \\
Q_{3} & =\left\{P: J<0,-J \leqslant \varepsilon<-\frac{2 J}{b}\right\}, & Q_{4} & =\left\{P: J<0, \frac{2 J}{b}<\varepsilon \leqslant \frac{J}{3}\right\}, \\
Q_{5} & =\left\{P: J<0,-\frac{2 J}{b} \leqslant \varepsilon<-\frac{J}{a}\right\}, & Q_{6} & =\left\{P: J<0, \frac{J}{a}<\varepsilon \leqslant \frac{2 J}{b}\right\}, \\
Q_{7} & =\left\{P: J<0, \varepsilon \geqslant-\frac{J}{a}\right\}, & Q_{8} & =\left\{P: J<0, \varepsilon \leqslant \frac{J}{a}\right\} .
\end{aligned}
$$

Tеорема 1. 1. Если $P \in Q_{1} \cup Q_{2}$, mо оператор $\widetilde{H}_{1}$ не имеет ЛПС.

2. Если $P \in Q_{3}\left(P \in Q_{4}\right)$, то оператор $\widetilde{H}_{1}$ имеет единственное ЛПС со значением энергии $z<m_{3}\left(z>M_{3}\right)$. Этот уровенъ энергии невырожден.

3. Если $P \in Q_{5}\left(P \in Q_{6}\right)$, то оператор $\widetilde{H}_{1}$ имеет ровно два ЛПС $\varphi_{1}$ и $\varphi_{2}$ со значениями энергии, равными соответственно $z_{1}$ u $z_{2}\left(z_{3} \quad u \quad z_{4}\right)$, причем $z_{i}<m_{3}$, 
$i=1,2\left(z_{j}>M_{3}, j=3,4\right)$. Уровень $z_{1}\left(z_{3}\right)$ невырожден, а уровень $z_{2}\left(z_{4}\right)$ двукратно вырожден.

4. Если $P \in Q_{7}\left(P \in Q_{8}\right)$, то оператор $\widetilde{H}_{1}$ имеет ровно три ЛПС $\varphi_{1}, \varphi_{2}$ и $\varphi_{3}$ со значениями энергии, равными соответственно $z_{1}, z_{2}$ и $z_{3}\left(z_{4}, z_{5}\right.$ u $\left.z_{6}\right)$, причем $z_{i}<m_{3}, i=1,2,3\left(z_{j}>M_{3}, j=4,5,6\right)$. Уровень $z_{1}\left(z_{4}\right)$ невырожден, уровенъ $z_{2}$ $\left(z_{5}\right)$ двукратно вырожден, а уровень $z_{3}\left(z_{6}\right)$ трехкратно вырожден.

ДокАЗАТЕЛЬСТво. Из формулы $(11)$ видно, что $\Delta_{3}(z)=0$, если верно хотя бы одно из нижеследующих равенств:

$$
\begin{aligned}
1-2 \varepsilon J_{3,1}(z) & =0 \\
{\left[1-2 \varepsilon J_{3,2}(z)\right]^{3} } & =0 \\
{\left[1-\varepsilon J_{3,3}(z)\right]^{2} } & =0 .
\end{aligned}
$$

Ясно, что функции

$$
\begin{aligned}
& J_{3,1}(z)=\int_{T^{3}} \frac{\left(1-\cos s_{1}\right)\left(3-\sum_{i=1}^{3} \cos s_{i}\right) d s_{1} d s_{2} d s_{3}}{h\left(s_{1} ; s_{2} ; s_{3}\right)-z}, \\
& J_{3,2}(z)=\int_{T^{3}} \frac{\sin ^{2} s_{1} d s_{1} d s_{2} d s_{3}}{h\left(s_{1} ; s_{2} ; s_{3}\right)-z} \\
& J_{3,3}(z)=\int_{T^{3}} \frac{\left(\cos s_{1}-\cos s_{2}\right)^{2} d s_{1} d s_{2} d s_{3}}{h\left(s_{1} ; s_{2} ; s_{3}\right)-z}
\end{aligned}
$$

являются монотонно возрастающими функциями $z$ для значений $z \notin\left[m_{3} ; M_{3}\right]$. Функция $J_{3,1}(z)$ при $z<m_{3}$ возрастает от 0 до $-1 /(2 J)$, а при $z>M_{3}-$ от $1 /(2 J)$ до 0. Кроме того, $J_{3,1}(z) \rightarrow 0$ при $z \rightarrow-\infty, J_{3,1}(z) \rightarrow-1 /(2 J)$ при $z \rightarrow m_{3}-0, J_{3,1}(z) \rightarrow$ $1 /(2 J)$ при $z \rightarrow M_{3}+0$ и $J_{3,1}(z) \rightarrow 0$ при $z \rightarrow \infty$. Поэтому функция $1-2 \varepsilon J_{3,1}(z)$ является монотонно убывающей функцией $z$ для значений $z<m_{3}$ при $\varepsilon>0$. Ясно, что $1-2 \varepsilon J_{3,1}(z) \rightarrow 1$ при $z \rightarrow-\infty$ и $1-2 \varepsilon J_{3,1}(z) \rightarrow 1+\varepsilon / J$ при $z \rightarrow m_{3}-0$. Поэтому уравнение (12) имеет решение при $z<m_{3}$, если $1+\varepsilon / J<0$, откуда мы получаем, что $\varepsilon>-J$. При условии, что $z>M_{3}$ и $\varepsilon<0$, функция $1-2 \varepsilon J_{3,1}(z)$ является монотонно возрастающей функцией $z$. Ясно, что $1-2 \varepsilon J_{3,1}(z) \rightarrow 1-\varepsilon / J$ при $z \rightarrow M_{3}+0$ и $1-2 \varepsilon J_{3,1}(z) \rightarrow 1$ при $z \rightarrow+\infty$. Поэтому уравнение $(12)$ имеет решение, если выполняется условие $\varepsilon<J$.

Функция $J_{3,2}(z)$ при $z<m_{3}$ возрастает от 0 до $-a /(2 J)$, а при $z>M_{3}$ - от $a /(2 J)$ до 0. Кроме того, $J_{3,2}(z) \rightarrow 0$ при $z \rightarrow-\infty, J_{3,2}(z) \rightarrow-a /(2 J)$ при $z \rightarrow m_{3}-0$, $J_{3,2}(z) \rightarrow a /(2 J)$ при $z \rightarrow M_{3}+0$ и $J_{3,2}(z) \rightarrow 0$ при $z \rightarrow \infty$. Поэтому функция $1-2 \varepsilon J_{3,2}(z)$ является монотонно убывающей функцией $z$ для значений $z<m_{3}$ и $\varepsilon>0$. Ясно, что $1-2 \varepsilon J_{3,2}(z) \rightarrow 1$ при $z \rightarrow-\infty$ и $1-2 \varepsilon J_{3,2}(z) \rightarrow 1+a \varepsilon / J$ при $z \rightarrow m_{3}-0$. Поэтому уравнение (13) имеет решение при $z<m_{3}$, если $1+a \varepsilon / J<0$. Отсюда мы получаем, что $\varepsilon>-J / a$. При $z>M_{3}$ уравнение (13) имеет решение, если выполняется условие $\varepsilon<J / a$. Аналогично функция $J_{3,3}(z)$ при $z<m_{3}$ возрастает от 0 до $-b /(2 J)$, а при $z>M_{3}-$ от $b /(2 J)$ до 0 . Кроме того, $J_{3,3}(z) \rightarrow 0$ при $z \rightarrow-\infty, J_{3,3}(z) \rightarrow-b /(2 J)$ при $z \rightarrow m_{3}-0, J_{3,3}(z) \rightarrow b /(2 J)$ при $z \rightarrow M_{3}+0$ 
и $J_{3,3}(z) \rightarrow 0$ при $z \rightarrow \infty$. Поэтому функция $1-\varepsilon J_{3,3}(z)$ является монотонно убывающей функцией $z$ для значений $z<m_{3}$ и $\varepsilon>0$. Ясно, что $1-\varepsilon J_{3,3}(z) \rightarrow 1$ при $z \rightarrow-\infty$ и $1-\varepsilon J_{3,3}(z) \rightarrow 1+b \varepsilon /(2 J)$ при $z \rightarrow m_{3}-0$. Поэтому уравнение (14) имеет решение при $z<m_{3}$, если $1+b \varepsilon /(2 J)<0$. Следовательно, $\varepsilon>-2 J / b$. При условии, что $z>M_{3}$, уравнение (14) имеет решение, если выполняется $\varepsilon<2 J / b$. Следовательно, при $z<m_{3}$ уравнение $\Delta_{3}(z)=0$ имеет три решения, если выполняются одновременно все эти три условия; имеет два решения, если выполняются два из этих условий одновременно. Если выполняется только одно из этих условий, то уравнение $\Delta_{3}(z)=0$ имеет единственное решение. Если ни одно из этих условий не выполняется, то уравнение $\Delta_{3}(z)=0$ не имеет решений. Аналогичная ситуация имеет место и при $z>M_{3}$. Кратность каждого решения определяется из вида функции $\Delta_{3}(z)$.

Обозначим через $a(z)$ интегральный оператор с ядром $h_{1}(x ; t) /(h(t)-z)$, а через $\mathrm{ext}_{1}$ и $\mathrm{ext}_{\mathrm{r}}$ - левую и правую крайние точки существенного спектра рассматриваемого трехчастичного оператора.

ОПрЕДЕЛЕНиЕ 2. Будем говорить, что оператор $\widetilde{H}_{1}$ имеет виртуальный уровень на краю существенного спектра $z=z_{\operatorname{ext}_{1}}\left(z=z_{\text {ext }_{\mathrm{r}}}\right)$, если число 1 является простым собственным значением оператора $a\left(z_{\text {ext }_{1}}\right)\left(a\left(z_{\mathrm{ext}_{\mathrm{r}}}\right)\right)$, причем для нетривиального решения уравнения $a\left(z_{\text {ext }_{1}}\right) \varphi=\varphi\left(a\left(z_{\text {ext }_{\mathrm{r}}}\right) \varphi=\varphi\right)$ выполнено условие $\varphi\left(z_{\text {ext }_{1}}\right) \neq 0$ $\left(\varphi\left(z_{\operatorname{ext}_{\mathrm{r}}}\right) \neq 0\right)$.

Отметим, что требование единственности (с точностью до множителя) решения уравнения $a\left(z_{\text {ext }_{1}}\right) \varphi=\varphi\left(a\left(z_{\text {ext }_{\mathrm{r}}}\right) \varphi=\varphi\right)$ и условие $\varphi\left(z_{\text {ext }_{1}}\right) \neq 0\left(\varphi\left(z_{\text {ext }_{\mathrm{r}}}\right) \neq 0\right)$ соответствуют отсутствию у оператора $\widetilde{H}_{1}$ собственных значений, paвных $z=z_{\text {ext }_{1}}$ $\left(z=z_{\text {ext }_{\mathrm{r}}}\right)$.

\section{3. ДВУХМАГНОННОЕ СОСТОЯНИЕ}

Гамильтониан двухмагнонной системы имеет вид

$$
H=J \sum_{m, \tau}\left(\vec{S}_{m} \vec{S}_{m+\tau}\right)
$$

где $J<0$ - параметр билинейного обменного взаимодействия между ближайшими атомами решетки. Гамильтониан (15) действует в симметрическом фоковском пространстве $\mathscr{H}$. Вектор $S_{m}^{-} S_{n}^{-} \varphi_{0}$ описывает состояние системы двух магнонов, находящихся в узлах $m$ и $n$ со значением спина $s=1 / 2$. Векторы $S_{m}^{-} S_{n}^{-} \varphi_{0}$ образуют ортонормальную систему. Пространство, натянутое на эти векторы, обозначим через $\widehat{\mathscr{H}}_{2}$. Оно является евклидовым относительно исходного скалярного произведения. Замыкание этого пространства обозначим через $\mathscr{H}_{2}$. Оно называется пространством двухмагнонных состояний оператора $H$.

ПрЕДЛОЖЕНИЕ 4. Пространство $\mathscr{H}_{2}$ инвариантно относительно оператора $H$. Оператор $\mathrm{H}_{2}=\left.H\right|_{\mathscr{H}_{2}}$ является ограниченным самосопряжсенным оператором, который порождает ограниченный самосопряженный оператор $\bar{H}_{2}$, действующий 
в пространстве $l_{2}\left(\mathbb{Z}^{3} \times \mathbb{Z}^{3}\right)$ по формуле

$$
\begin{aligned}
\left(\bar{H}_{2} f\right)(p ; q)=J & \sum_{p, q, \tau}\left\{\left(\delta_{p, q+\tau}+\delta_{p+\tau, q}-2\right) f(p ; q)-\frac{1}{2} \delta_{p-\tau, q} f(p-\tau ; q)-\right. \\
& -\frac{1}{2} \delta_{p, q-\tau} f(p ; q-\tau)+\frac{1}{2} f(p-\tau ; q)+ \\
& +\frac{1}{2} f(p ; q-\tau)-\frac{1}{2} \delta_{p+\tau, q} f(p+\tau ; q)- \\
& \left.-\frac{1}{2} \delta_{p, q+\tau} f(p ; q+\tau)+\frac{1}{2} f(p+\tau ; q)+\frac{1}{2} f(p ; q+\tau)\right\}
\end{aligned}
$$

где $\delta_{k, j}$ - символ Кронекера. Сам оператор $\mathrm{H}_{2}$ действует на вектор $\psi \in \mathscr{H}_{2}$ по формуле

$$
H_{2}=\sum_{p, q, \tau}\left(\bar{H}_{2} f\right)(p ; q) S_{p}^{-} S_{q}^{-} \varphi_{0}
$$

Предложение 4 доказывается с использованием известных коммутационных соотношений между операторами $S_{m}^{+}, S_{p}^{-}$и $S_{q}^{z}$ :

$$
\left[S_{m}^{+}, S_{p}^{-}\right]=2 \delta_{m, p} S_{m}^{z}, \quad\left[S_{p}^{ \pm}, S_{q}^{z}\right]= \pm \delta_{p, q} S_{p}^{ \pm}
$$

Используя критерий Вейля [22], можно установить, что спектры операторов $H_{2}$ и $\bar{H}_{2}$ совпадают.

Оператор (15) в квазиимпульсном представлении в пространстве $L_{2}\left(T^{\nu} \times T^{\nu}\right)$ действует по формуле

$$
\left(\widetilde{H}_{2} f\right)(x ; y)=h(x ; y) f(x ; y)-\int_{T^{3}} h_{1}(x ; y ; s) f(s ; x+y-s) d s
$$

где $h_{1}(x ; y ; s)=h(x ; y)+h_{2}(x ; y ; s)$. Здесь

$$
h(x ; y)=-12 J+2 J \sum_{i=1}^{3}\left(\cos x_{i}+\cos y_{i}\right)
$$

и

$$
h_{2}(x ; y ; s)=2 J \sum_{i=1}^{3}\left[\cos s_{i}+\cos \left(x_{i}+y_{i}-s_{i}\right)-\cos \left(x_{i}-s_{i}\right)-\cos \left(y_{i}-s_{i}\right)\right] .
$$

Спектр и связанные состояния оператора энергии двухмагнонных систем были изучены в работах [23], [24]. Пусть $\widetilde{\mathscr{H}}_{2}=\mathscr{F}_{2}$. Рассмотрим многообразие $\Gamma_{\Lambda}=\left\{(x ; y): x, y \in T^{\nu}, x+y=\Lambda\right\}$. Следующий факт является важным для последующего исследования спектра оператора $\widetilde{H}_{2}$. Пусть фиксирован полный квазиимпульс системы $x+y=\Lambda$. Обозначим через $L_{2}\left(\Gamma_{\Lambda}\right)$ пространство функций, квадратично-интегрируемых по многообразию $\Gamma_{\Lambda}=\{(x ; y): x+y=\Lambda\}$. Известно [25], что оператор $\widetilde{H}_{2}$ и пространство $\widetilde{\mathscr{H}}_{2}$ можно разложить в прямой интеграл $\widetilde{H}_{2}=\bigoplus \int_{T^{3}} \widetilde{H}_{2 \Lambda} d \Lambda, \widetilde{\mathscr{H}}_{2}=\bigoplus \int_{T^{3}} \widetilde{\mathscr{H}}_{2 \Lambda} d \Lambda$ операторов $\widetilde{H}_{2 \Lambda}$ и пространств $\widetilde{\mathscr{H}}_{2 \Lambda}=$ 
$L_{2}\left(\Gamma_{\Lambda}\right)$ так, что пространства $\widetilde{\mathscr{H}}_{2 \Lambda}$ окажутся инвариантными относительно операторов $\widetilde{H}_{2 \Lambda}$, а операторы $\widetilde{H}_{2 \Lambda}$ в пространстве $\widetilde{\mathscr{H}}_{2 \Lambda}$ действуют по формуле

$$
\left(\widetilde{H}_{2 \Lambda} f_{\Lambda}\right)(x)=h_{\Lambda}(x) f_{\Lambda}(x)-\int_{T^{3}} h_{1 \Lambda}(x ; t) f_{\Lambda}(t) d t,
$$

где $h_{\Lambda}(x)=h(x ; \Lambda-x), h_{1 \Lambda}(x ; t)=h_{1}(x ; \Lambda-x ; t)$ и $f_{\Lambda}(x)=f(x ; \Lambda-x)$.

Известно также, что непрерывный спектр оператора $\widetilde{H}_{2 \Lambda}$ не зависит от функций $h_{1 \Lambda}(x ; t)$ и состоит из отрезков $G_{\Lambda}=\left[m_{\Lambda} ; M_{\Lambda}\right]$, где $m_{\Lambda}=\inf _{x} h_{\Lambda}(x)$ и $M_{\Lambda}=$ $\sup _{x} h_{\Lambda}(x)$.

ОПредЕЛЕниЕ 3 . Собственная функция $\varphi \in L_{2}\left(T^{3} \times T^{3}\right)$ оператора $\widetilde{H}_{2}$, отвечающая собственному значению $z \notin G_{\Lambda}$, называется СС оператора $\widetilde{H}_{2}$, а величина $z-$ энергией этого состояния.

\section{4. СТРУКТУРА СУЩЕСТВЕННОГО СПЕКТРА ТРЕХЧАСТИЧНОЙ СИСТЕМЫ}

Определим сначала структуру существенного спектра рассматриваемой трехчастичной системы, а затем проверим, сколько двухчастичных подсистем данной системы имеют виртуальные уровни на краю существенного спектра.

Сравнивая формулы (7) и (10) и используя тензорные произведения гильбертовых пространств и тензорные произведения операторов в гильбертовых пространствах [22], можно убедиться, что оператор $\widetilde{H}_{2}$ можно представить в виде $\widetilde{H}_{2}=$ $\widetilde{H}_{1} \otimes E+E \otimes \widetilde{H}_{1}+K_{1}+K_{2}$, где $E-$ единичный оператор в пространстве $\widetilde{\mathscr{H}}_{1}$, а операторы $K_{1}$ и $K_{2}$ имеют следующий вид:

$$
\begin{aligned}
K_{1}(x ; y ; s)=J & \int_{T^{3}}\left\{12-\sum_{i=1}^{3}\left[\cos s_{i}+\cos x_{i}+\cos y_{i}+\right.\right. \\
& \left.\left.+\cos \left(x_{i}+y_{i}-s_{i}\right)\right]\right\} f(s ; x+y-s) d s \\
K_{2}(x ; y ; s ; t)=\varepsilon & \int_{T^{3}} \int_{T^{3}}\left\{6+2 \sum_{i=1}^{3}\left[\cos \left(x_{i}-s_{i}\right)+\cos \left(y_{i}-t_{i}\right)+\right.\right. \\
& \left.+\cos \left(x_{i}+y_{i}-s_{i}-t_{i}\right)\right]-\cos t_{i}-\cos s_{i}- \\
& -\cos x_{i}-\cos y_{i}-\cos \left(x_{i}-s_{i}-t_{i}\right)-\cos \left(y_{i}-s_{i}-t_{i}\right)- \\
& \left.-\cos \left(x_{i}+y_{i}-t_{i}\right)-\cos \left(x_{i}+y_{i}-s_{i}\right)\right\} f(s ; t) d s d t,
\end{aligned}
$$

где $\varepsilon=J_{0}-J$.

Рассматриваемая трехчастичная система является замкнутой, а в замкнутых системах имеет место закон сохранения полного квазиимпульса системы. Кроме того, примесь закреплена в определенном узле (в узле с номером $m=0$ ), поэтому полный квазиимпульс трехчастичной системы и полный квазиимпульс двухмагнонной подсистемы равны. Теперь, фиксируя полный квазиимпульс системы, можно разложить в прямой интеграл оператор $\widetilde{H}_{2}$ и пространство $\widetilde{\mathscr{H}}_{2}$ так, что после разложения 
в прямой интеграл операторы $K_{1 \Lambda}$ и $K_{2 \Lambda}$ становятся компактными. Поэтому существенный спектр оператора $\widetilde{H}_{2}$ и оператора $\widetilde{H}_{1} \otimes E+E \otimes \widetilde{H}_{1}$ совпадают. Простая проверка показывает, что спектр оператора $\widetilde{H}_{1}$ не зависит от $\Lambda$. Спектр оператора $A \otimes E+E \otimes B$, где $A$ и $B$ - плотно определенные ограниченные линейные операторы, был изучен в работах [26]-[28]. В этих работах даны явные формулы, выражающие $\sigma_{\text {ess }}(A \otimes E+E \otimes B)$ через $\sigma(A), \sigma_{\text {disc }}(A), \sigma(B)$ и $\sigma_{\text {disc }}(B)$. Ясно, что $\sigma(A \otimes E+E \otimes B)=\{\lambda+\mu: \lambda \in \sigma(A), \mu \in \sigma(B)\}$. Из полученных результатов видно, что спектр оператора $\widetilde{H}_{1}$ состоит из непрерывного спектра и не более чем трех собственных значений: невырожденного, двукратно вырожденного и трехкратно вырожденного.

Следующая теорема описывает структуру существенного спектра оператора $\widetilde{H}_{1} \otimes$ $E+E \otimes \widetilde{H}_{1}$.

Теорема 2. Если $0<\varepsilon<-J$ или $J / 3<\varepsilon<0$, то существенный спектр оператора $\widetilde{H}_{2}$ coстоит из единственного отрезка $G_{1}=[0 ;-24 J]$, m.e. $\sigma_{\mathrm{ess}}\left(\widetilde{H}_{2}\right)=$ $[0 ;-24 J]$ и дискретный спектр оператора конечен.

ДоказАтельство. Из теоремы 1 видно, что для $0<\varepsilon<-J$ или $J / 3<\varepsilon<0$ оператор $\widetilde{H}_{1}$ не имеет собственных значений, лежащих вне области непрерывного спектра оператора $\widetilde{H}_{1}$. А непрерывный спектр оператора $\widetilde{H}_{1}$ состоит из отрезка $[0 ;-12 J]$, поэтому существенный спектр оператора $\widetilde{H}_{2}$ состоит из отрезка $[0 ;-24 J]$, т.е. $\sigma_{\text {ess }}\left(\widetilde{H}_{2}\right)=\left\{\lambda+\mu: \lambda \in \sigma\left(\widetilde{H}_{1}\right), \mu \in \sigma\left(\widetilde{H}_{1}\right\}=[0 ;-24 J]\right.$. Покажем, что оператор $\widetilde{H}_{1}$ в точках $z=0$ и $z=-24 J$ не имеет виртуальных уровней. Оператор $a(0)$ имеет простое собственное значение, равное 1 (см. формулу (11)), только при $J_{0}=0$, а поскольку $J_{0} \neq 0$, то оператор $\widetilde{H}_{1}$ в точке $z=0$ не имеет виртуального уровня. $\mathrm{B}$ точке $z=0$ две двухчастичные подсистемы не имеют виртуальных уровней, поэтому дискретный спектр трехчастичного оператора на краю существенного спектра $z=0$ конечен. Теперь покажем, что в точке $z=-24 J$ оператор $\widetilde{H}_{1}$ также не имеет виртуального уровня. Для того чтобы оператор $a(-24 J)$ имел простое собственное значение, равное 1 (см. формулу (11)), должно выполняться равенство

$$
1-\frac{\varepsilon}{J} \int_{T^{3}} \frac{\left(1-\cos s_{1}\right)\left(3-\cos s_{1}-\cos s_{2}-\cos s_{3}\right)}{9+\cos s_{1}+\cos s_{2}+\cos s_{3}} d s_{1} d s_{2} d s_{3}=0 .
$$

Это соотношение верно, если $\varepsilon=J /(48 c-5)$, где

$$
c=\int_{T^{3}} \frac{d s_{1} d s_{2} d s_{3}}{9+\cos s_{1}+\cos s_{2}+\cos s_{3}} .
$$

Проверка показывает, что $5 / 48<c<1 / 6$, но такие пары $(J ; \varepsilon)$ не принадлежат множеству значений $0<\varepsilon<-J$ и $J / 3<\varepsilon<0$, следовательно, в точке $z=-24 J$ оператор $\widetilde{H}_{1}$ не имеет виртуального уровня.

Теорема 3. Если $-J \leqslant \varepsilon<-2 J / b$ или $2 J / b \leqslant \varepsilon \leqslant J / 3$, то существенный спектр оператора $\widetilde{H}_{2}$ состоит из обгединения двух отрезков: $\sigma_{\mathrm{ess}}\left(\widetilde{H}_{2}\right)=[0 ;-24 J] \cup$ $\left[z_{1} ;-12 J+z_{1}\right]$, и на краях существенного спектра дискретный спектр оператора $\widetilde{H}_{2}$ конечен. 
ДокАЗАТЕЛЬСтво. Из теоремы 1 видно, что для $2 J / b \leqslant \varepsilon \leqslant J / 3$ или $-J \leqslant$ $\varepsilon<-2 J / b$ оператор $\widetilde{H}_{1}$ имеет единственное собственное значение $z=z_{1}$, лежащее вне области непрерывного спектра оператора $\widetilde{H}_{1}$. Поэтому множество $\{\lambda+\mu: \lambda \in$ $\sigma\left(\widetilde{H}_{1}\right), \mu \in \sigma\left(\widetilde{H}_{1}\right\}$ состоит из объединения двух отрезков $[0 ;-24 J]$ и $\left[z_{1} ;-12 J+z_{1}\right]$, т.е. $\sigma_{\text {ess }}\left(\widetilde{H}_{2}\right)=[0 ;-24 J] \cup\left[z_{1} ;-12 J+z_{1}\right]$. Если $-J \leqslant \varepsilon<-2 J / b$, то $z_{1}<0$, т.е. собственное значение оператора $\widetilde{H}_{1}$ лежит ниже области непрерывного спектра оператора $\widetilde{H}_{1}$, если же $2 J / b \leqslant \varepsilon \leqslant J / 3$, то $z_{1}>-12 J$, т.е. собственное значение оператоpa $\widetilde{H}_{1}$ лежит выше области непрерывного спектра оператора $\widetilde{H}_{1}$. В первом случае нижним краем существенного спектра рассматриваемой трехчастичной системы является точка $z=z_{1}$, и она является собственным значением оператора $\widetilde{H}_{1}$, поэтому точка $z=z_{1}$ не может быть виртуальным уровнем для оператора $\widetilde{H}_{1}$. Верхним краем является точка $z=-24 J$. В предыдущей теореме показано, что точка $z=-24 J$ не может быть виртуальным уровнем для оператора $\widetilde{H}_{1}$. Это означает, что дискретный спектр оператора $\widetilde{H}_{2}$ конечен. Во втором случае точка $z=0$ является нижним краем существенного спектра и поэтому не может быть виртуальным уровнем для оператора $\widetilde{H}_{1}$. Точка $z=-12 J+z_{1}$ является верхним краем существенного спектра трехчастичной системы. Покажем, что эта точка также не может быть виртуальным уровнем оператора $\widetilde{H}_{1}$. Если точка $z=-12 J+z_{1}$ является виртуальным уровнем оператора $\widetilde{H}_{1}$, то

$$
1-2 \varepsilon \int_{T^{3}} \frac{\left(1-\cos s_{1}\right)\left(3-\sum_{i=1}^{3} \cos s_{i}\right)}{-2 J\left(3-\sum_{i=1}^{3} \cos s_{i}\right)+12 J-z_{1}} d s_{1} d s_{2} d s_{3}=0 .
$$

Уравнение (19) эквивалентно уравнению вида

$$
1+\frac{\varepsilon}{J}-\frac{\varepsilon\left(z_{1}-12 J\right)}{6 J^{2}}-\frac{\varepsilon\left(z_{1}-12 J\right)^{2}}{12 J^{3}} d=0,
$$

где

$$
d=\int_{T^{3}} \frac{d s_{1} d s_{2} d s_{3}}{3+\sum_{i=1}^{3} \cos s_{i}-z_{1} /(2 J)}
$$

Отсюда находим, что

$$
\varepsilon=-\frac{12 J^{3}}{12 J^{2}+2 J\left(z_{1}-12 J\right)-\left(z_{1}-12 J\right)^{2} d} .
$$

Для того чтобы пара $(J ; \varepsilon)$ принадлежала рассматриваемой области, $d$ должно быть отрицательным числом, но на самом деле $d>0$. Поэтому точка $z=-12 J+z_{1}$ не может быть виртуальным уровнем оператора $\widetilde{H}_{1}$. Следовательно, в этом случае дискретный спектр рассматриваемого трехчастичного оператора на краях существенного спектра конечен.

Теорема 4. Если $-2 J / b \leqslant \varepsilon<-J / a$ или $J / a \leqslant \varepsilon \leqslant 2 J / b$, то существенный спектр оператора $\widetilde{H}_{2}$ состоит из обгединения трех отрезков: $\sigma_{\mathrm{ess}}\left(\widetilde{H}_{2}\right)=$ $[0 ;-24 J] \cup\left[z_{1} ;-12 J+z_{1}\right] \cup\left[z_{2} ;-12 J+z_{2}\right]$, и на краях существенного спектра дискретный спектр оператора $\widetilde{H}_{2}$ конечен. 
ДокАЗАтельство. Если $-2 J / b \leqslant \varepsilon<-J / a$, то оператор $\widetilde{H}_{1}$ имеет ровно два собственных значения: $z=z_{1}$ и $z=z_{2}$, которые лежат ниже области непрерывного спектра оператора $\widetilde{H}_{1}$, т.е. $z_{i}<0, i=1,2$. Пусть $z_{1}<z_{2}$, тогда точка $z=z_{1}$ является нижним краем существенного спектра трехчастичной системы. Но в этой точке оператор $\widetilde{H}_{1}$ не может иметь виртуального уровня, так как точка $z=z_{1}$ является собственным значением для оператора $\widetilde{H}_{1}$. Верхним краем существенного спектра является точка $z=-24 J$. Ранее показано, что в точке $z=-24 J$ оператор $\widetilde{H}_{1}$ не имеет виртуального уровня. Если же $J / a \leqslant \varepsilon \leqslant 2 J / b$, то оператор $\widetilde{H}_{1}$ имеет ровно два собственных значения: $z=z_{1}$ и $z=z_{2}$, которые лежат выше области непрерывного спектра оператора $\widetilde{H}_{1}$, т.е. $z_{i}>-12 J, i=1,2$. Пусть $z_{1}>z_{2}$. Тогда точка $z=0$ является нижним краем существенного спектра трехчастичного оператора, поэтому в точке $z=0$ оператор $\widetilde{H}_{1}$ не имеет виртуального уровня. Верхним краем существенного спектра трехчастичного оператора является точка $z=-12 J+z_{1}$. В этой точке оператор $\widetilde{H}_{1}$ не может иметь виртуального уровня, так как в рассматриваемых множествах значений пар $(J ; \varepsilon)$ оператор $a\left(z=-12 J+z_{1}\right)$ не имеет простого собственного значения, равного 1 . Если оператор $a\left(z=-12 J+z_{1}\right)$ в этих множествах имеет собственное значение, то это собственное значение будет кратным. Поэтому в точке $z=-12 J+z_{1}$ оператор $\widetilde{H}_{1}$ не имеет виртуального уровня. Следовательно, дискретный спектр трехчастичного оператора в этих точках конечен.

ТеОрема 5. Если $\varepsilon \geqslant-J / a$ или $\varepsilon \leqslant J / a$, то существенный спектр оператоpa $\widetilde{H}_{2}$ состоит из обгединения четырех отрезков: $\sigma_{\mathrm{ess}}\left(\widetilde{H}_{2}\right)=[0 ;-24 J] \cup\left[z_{1} ;-12 J+\right.$ $\left.z_{1}\right] \cup\left[z_{2} ;-12 J+z_{2}\right] \cup\left[z_{3} ;-12 J+z_{3}\right]$, и на краях существенного спектра дискретньй спектр оператора $\widetilde{H}_{2}$ конечен.

ДокАЗАТЕЛьСтво теоремы 5 повторяет доказательство теоремы 4.

\section{Список литературы}

[1] В. Н. Ефимов, ЯФ, 12:5 (1970), 1080-1091.

[2] R. D. Amado, J. V. Noble, Phys. Lett. B, 35:1 (1971), 25-27.

[3] R. D. Amado, J. V. Noble, Phys. Rev. D, 5:8 (1972), 1992-2002.

[4] Д. Р. Яфаев, Матем. сб., 94(136):4(8) (1974), 567-593.

[5] Yu. N. Ovchinnikov, J. M. Sigal, Ann. Physics, 123:2 (1989), 274-295.

[6] H. Tamura, J. Funct. Anal., 95:2 (1991), 433-459.

[7] A. V. Sobolev, Comm. Math. Phys., 156:1 (1993), 101-126.

[8] D. C. Mattis, Rev. Modern Phys., 58:2 (1986), 361-379.

[9] В. А. Малышев, Р. А. Минлос, "Кластерные операторы”, Труды семинара им. И. Г. Петровского, 9, 1983, 63-80.

[10] A. I. Mogilner, "The problem of few quasi particles in solid state Physics", Applications of Self-Adjoint Extentions in Quantum Particles, Lecture Notes in Phys., 324, eds. P. Exner, P. Šeba, Springer, Berlin, 1989, 160-173.

[11] С. Н. Лакаев, Функи. анализ и его прил., 27:3 (1993), 15-28.

[12] С. Н. Лакаев, ТМФ, 89:1 (1991), 94-104.

[13] С. Н. Лакаев, ДАН Уз. ССР, 1991, № 6, 11-14.

[14] С. Н. Лакаев, С. М. Саматов, ТМФ, 129:3 (2001), 415-431.

[15] Ю. А. Изюмов, М. В. Медведев, ЖЭЭТФ, 48:2 (1965), 574-586. 
[16] В. В. Ганн, Л. Г. Зазунов, Физика твердого тела, 15:12 (1973), 3535-3569.

[17] Wang Yung-Li, H. Callen, Phys. Rev., 160:2 (1967), 358-363.

[18] T. Ogychi, I. Ono, J. Phys. Soc. Japan, 26 (1969), 32-42.

[19] T. Wolfram, J. Callaway, Phys. Rev., 130:6 (1963), 2207-2217.

[20] I. Ono, Y. Endo, Phys. Lett. A, 41:5 (1972), 440-442.

[21] С. М. Ташпулатов, ТМФ, 126:3 (2001), 482-488.

[22] М. Рид, Б. Саймон, Методы современной математической физики. Т. 1. Функииональный анализ, Мир, М., 1977.

[23] M. Wortis, Phys. Rev., 132:1 (1963), 85-97.

[24] С. М. Ташпулатов, ТМФ, 107:1 (1996), 155-161.

[25] М. А. Наймарк, Нормированные колъца, Наука, М., 1968.

[26] T. Ichinose, Trans. Amer. Math. Soc., 235 (1978), 75-113.

[27] T. Ichinose, Trans. Amer. Math. Soc., 237 (1978), 223-254.

[28] T. Ichinose, "On the spectral properties of tensor products of linear operators in Banach spaces", Spectral Theory, Banach Center Publ., 8, PWN, Warsaw, 1982, 295-300.

Поступила в редакцию 2.09.2008, после доработки 10.04.2009 